\title{
Asynchronously Evolving Solutions with Excessively Different Evaluation Time by Reference-based Evaluation
}

2014/07/15

\section{Tomohiro Harada ${ }^{1,2}$, Keiki Takadama ${ }^{1}$}

${ }^{1}$ The University of Electro-Communications, Japan ${ }^{2}$ Research Fellow of the Japan Society for the Promotion of Science DC1, Japan 


\section{Introduction}

\section{Synchronous EA and its problem}

General EA=Synchronous EA

evolves individuals depending on evaluations of entire population

If evaluation time of individuals differs from each other

$\rightarrow$ sync. EA needs to wait for the slowest one

e.g., $(\mu+\lambda)$-selection
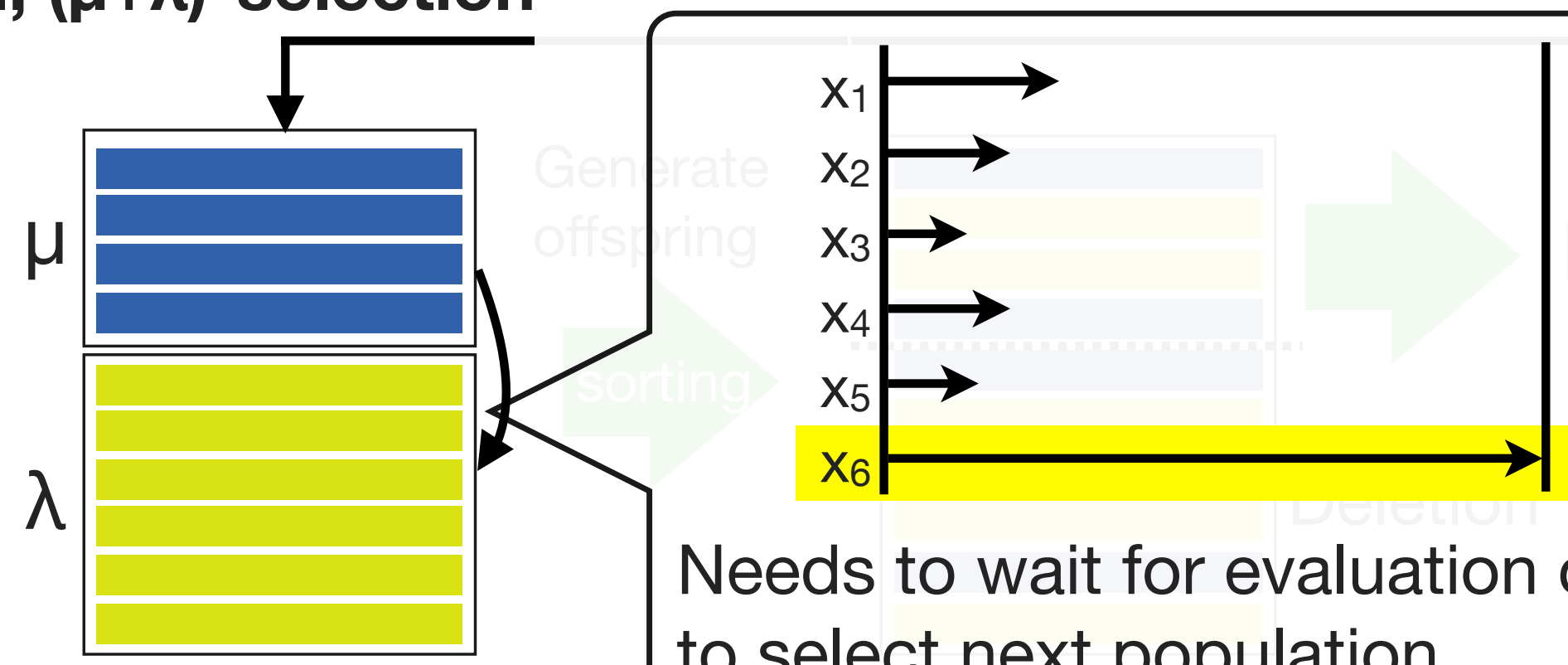

Needs to wait for evaluation of $\mathrm{X}_{6}$

Evaluate to select next population all individuals 


\section{Asynchronous EA}

evolves individuals independently (asynchronously)

e.g., TAGP [Harada2013]
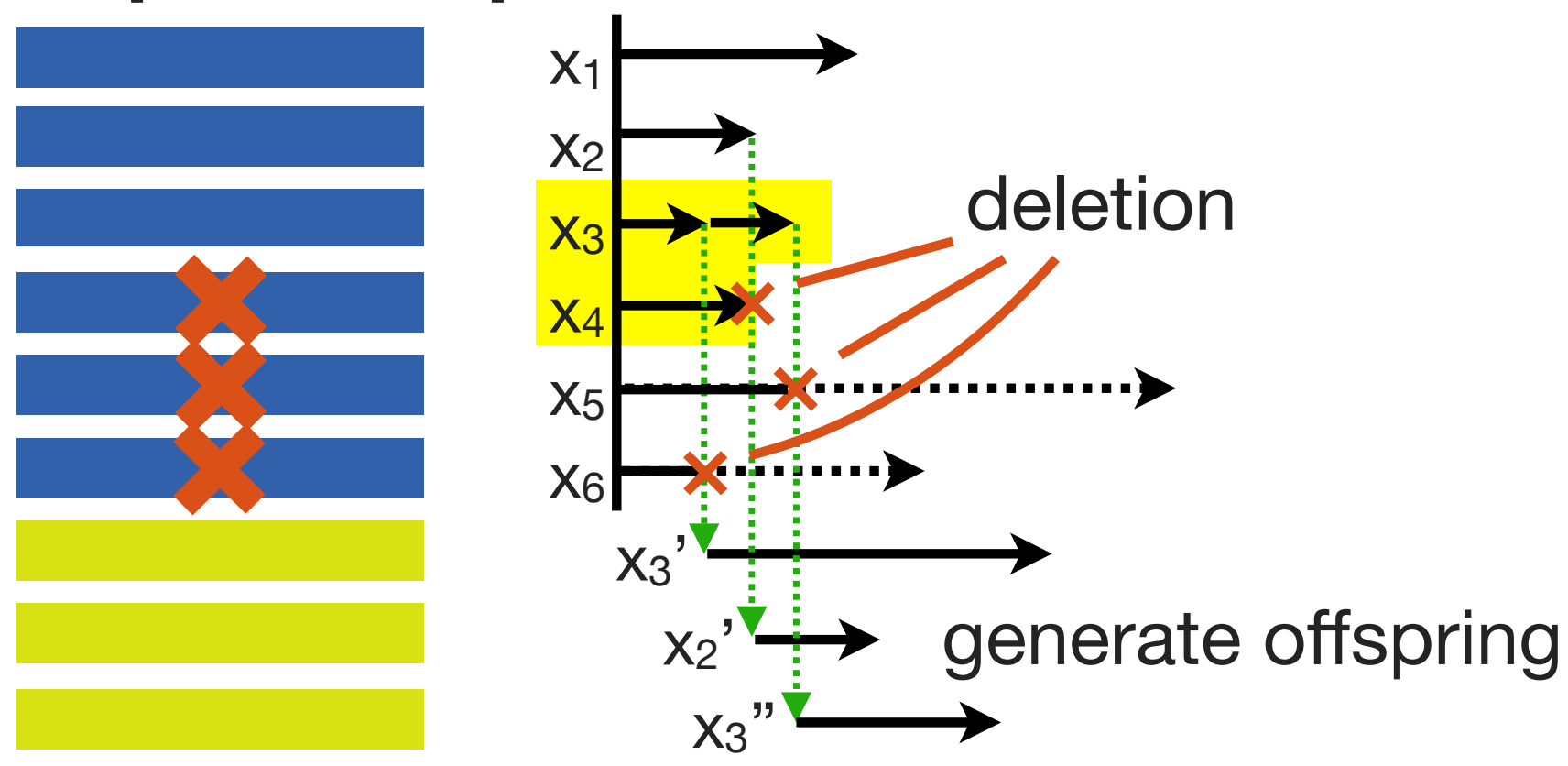

Advantage : Async. EA needs not to wait for other individuals $\rightarrow$ can continue an evolution even in different evaluation time 


\section{Difficulties of Async. EA}

1. How to preserve good individuals?

2. How to delete bad individuals?

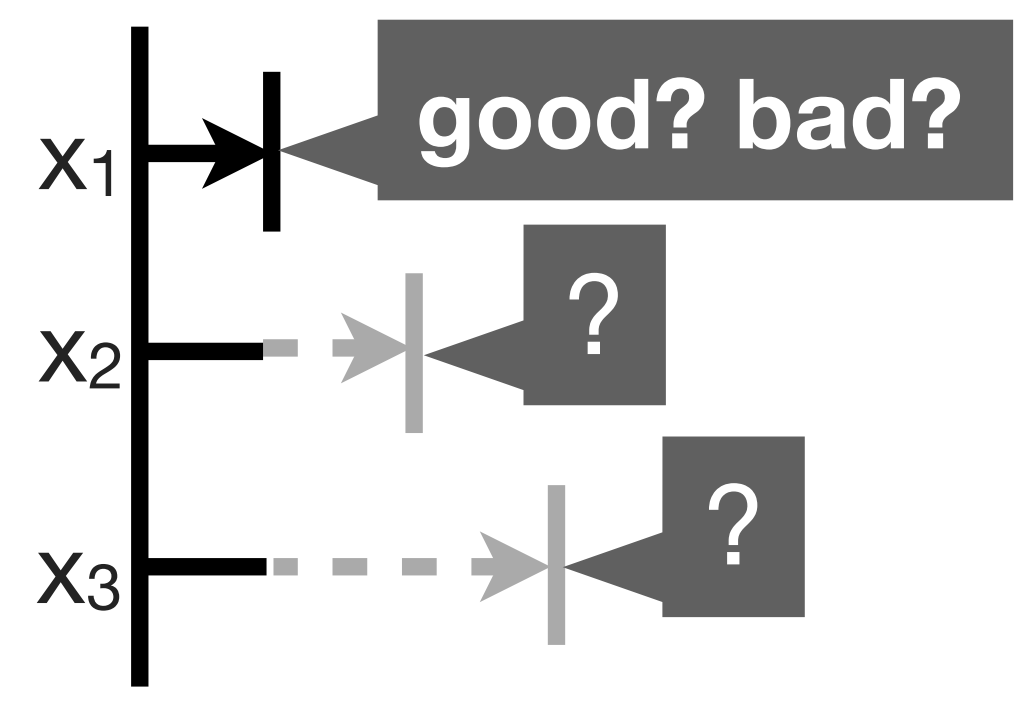




\section{Objective}

To propose EA using Asynchronous Reference-based Evaluation (ARE-EA)

- Archive mechanism to preserve good individuals

- Reference individual to delete bad individuals

To investigate effectiveness of ARE-EA in situation where evaluation times are excessively different

1. Different computing speed (e.g., Difference of processing ability)

2. Evaluation failure 


\section{Proposed method ARE-EA}

\section{Archive}

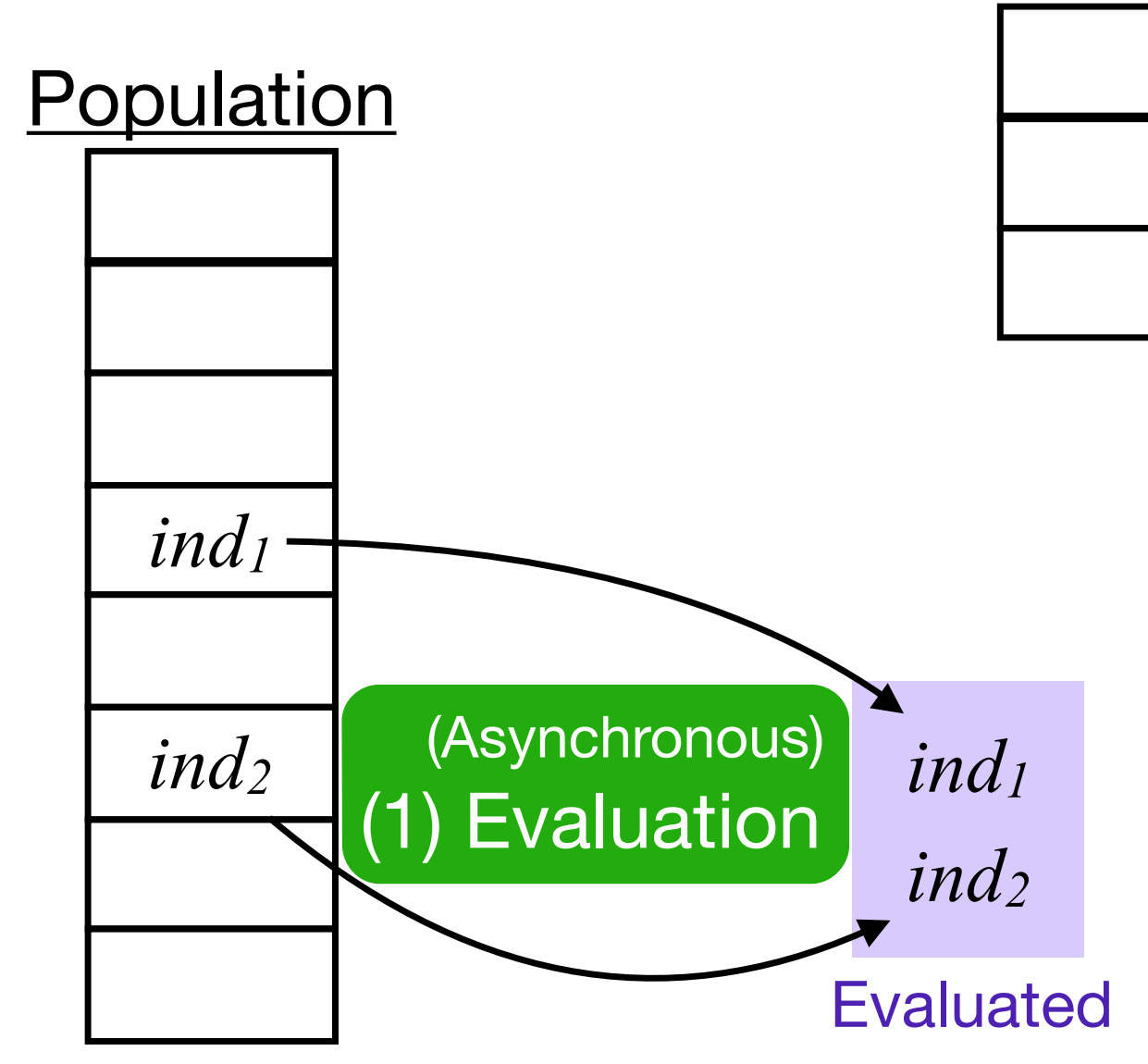

Archive size 


\section{Proposed method ARE-EA}

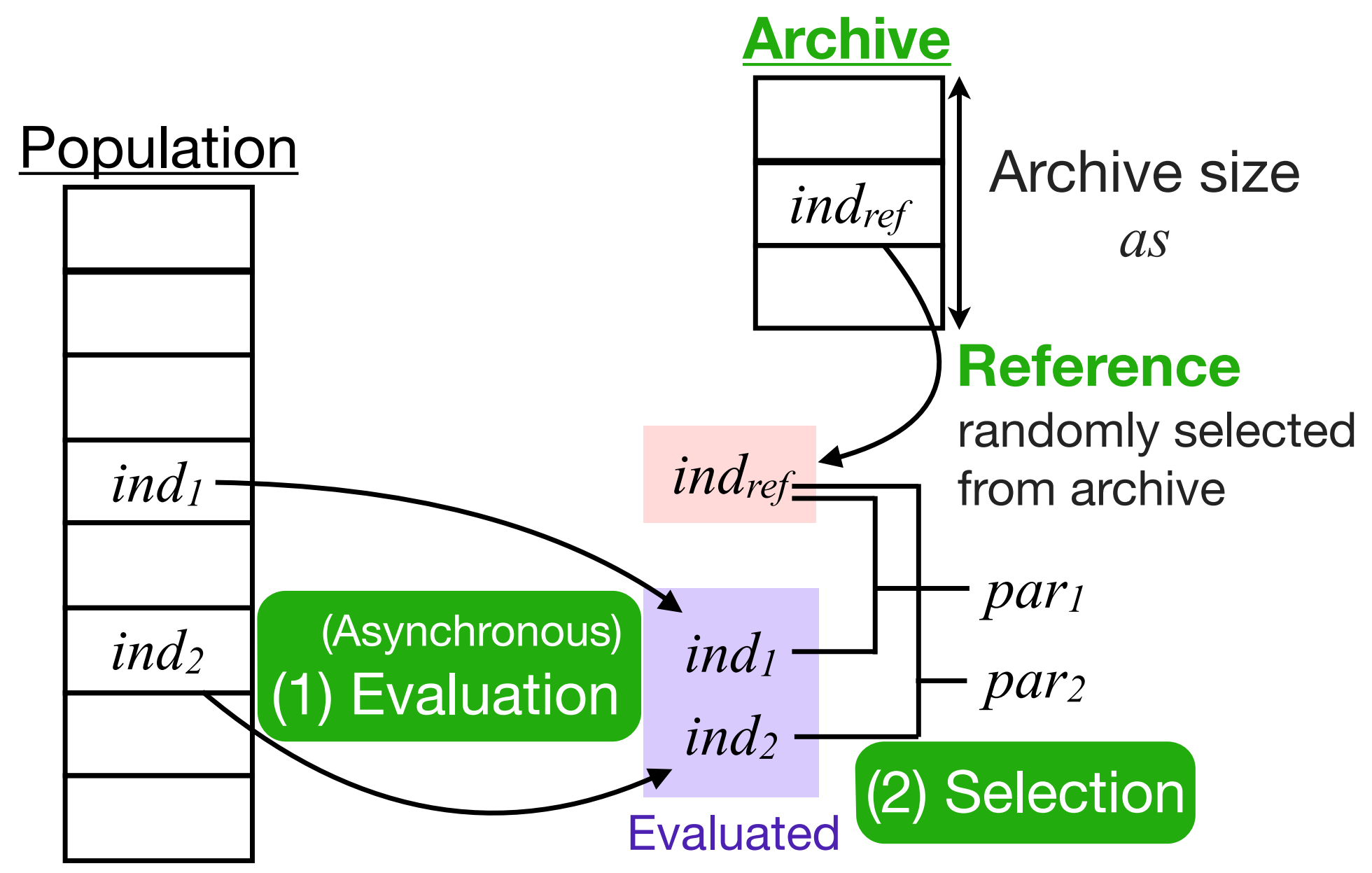

Good individuals (indref) are utilized for selection 


\section{Proposed method ARE-EA}

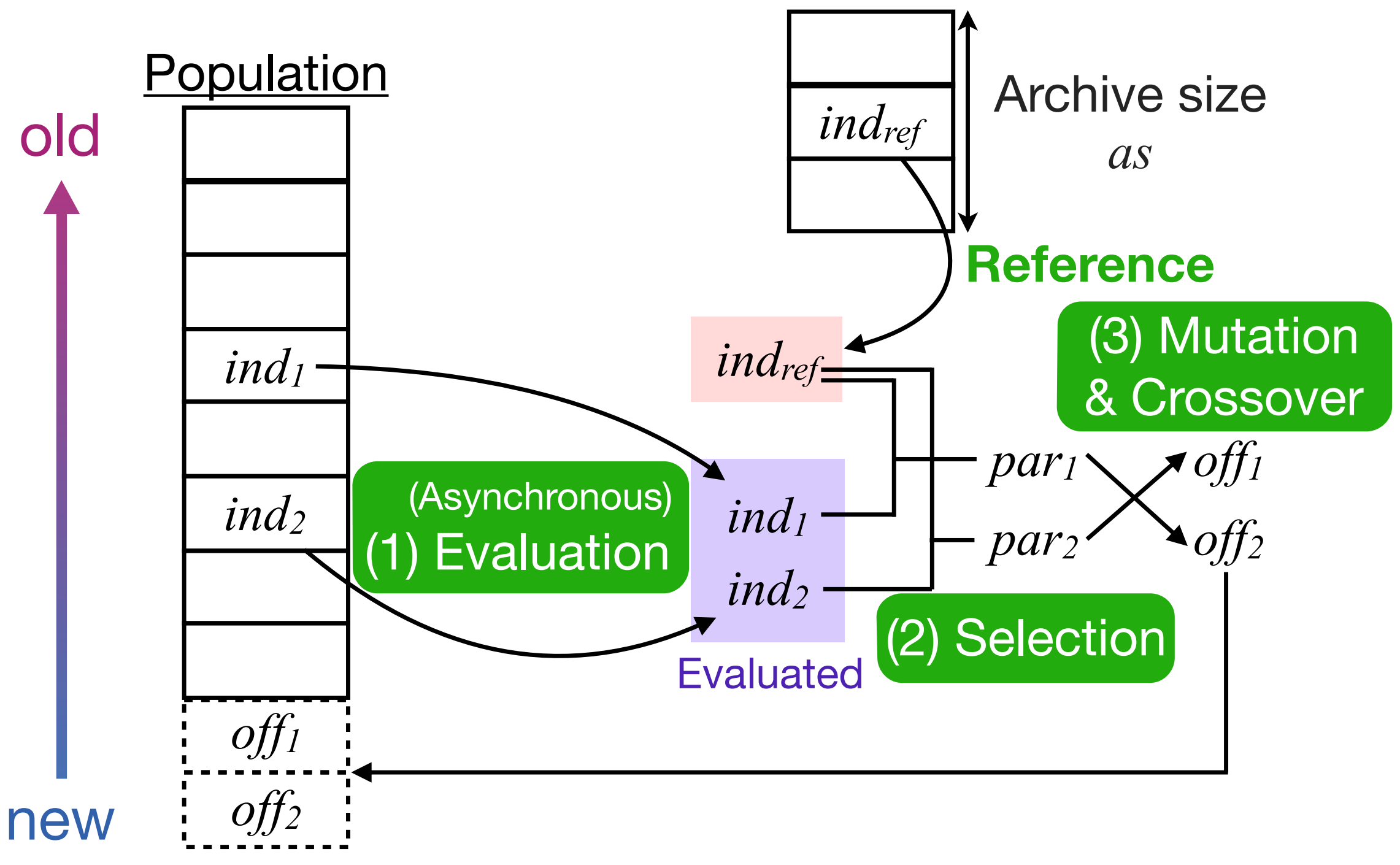




\section{Proposed method ARE-EA}

\section{(4) Archiving}

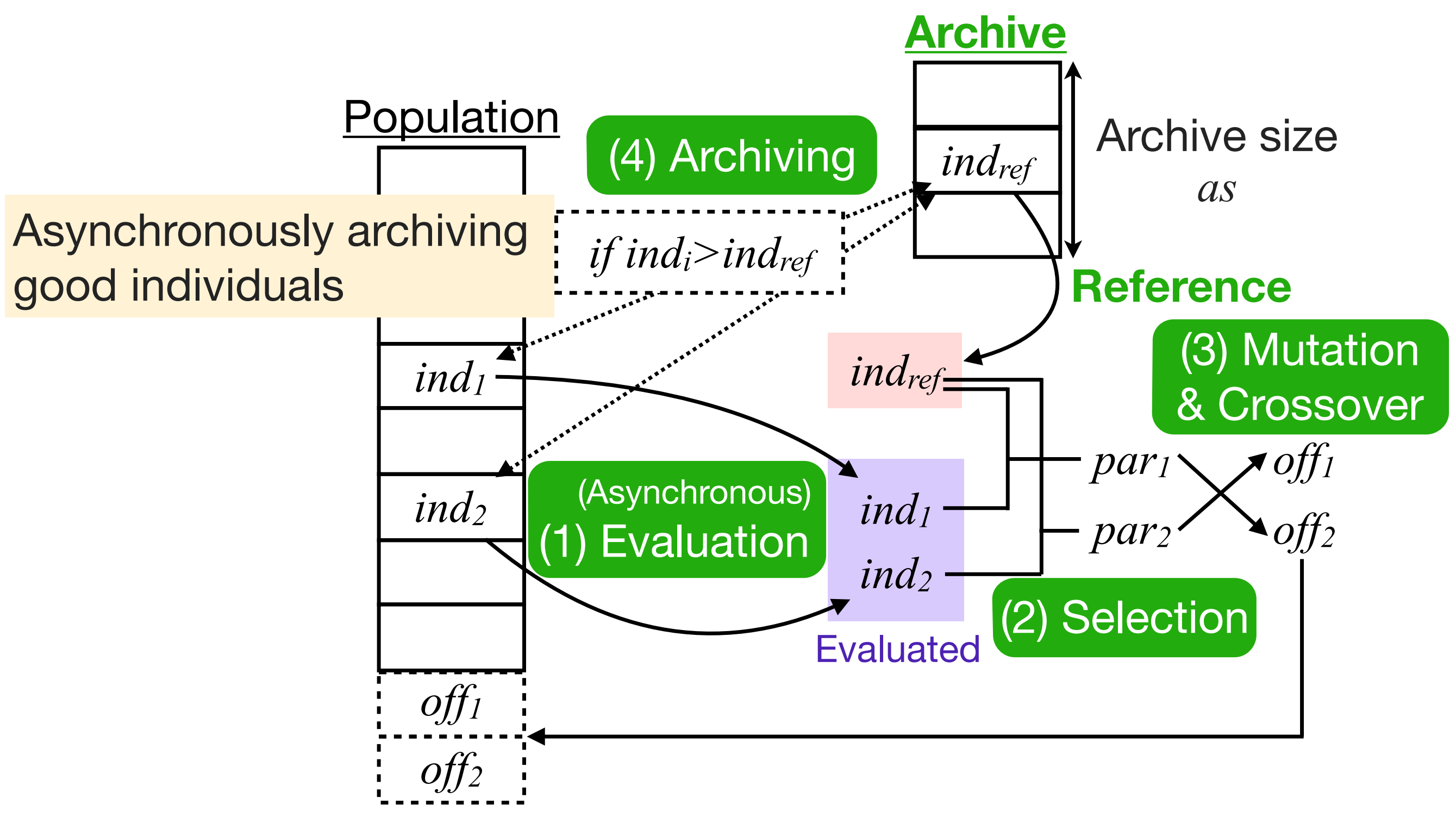




\section{Proposed method ARE-EA}

(5) Fitness deletion
(6) Reaper deletion

(6) Reaper deletion

(5) Fitness deletion

if ind ${ }_{i}<$ ind $_{\text {ref }}$ with probability $P_{d}$

\section{Population}
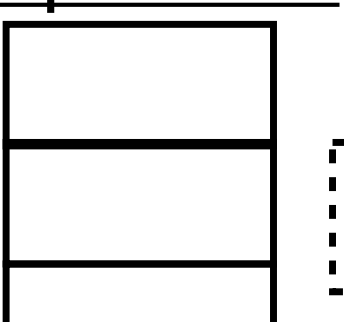

(4) Archiving

if ind ${ }_{i}>$ ind ref
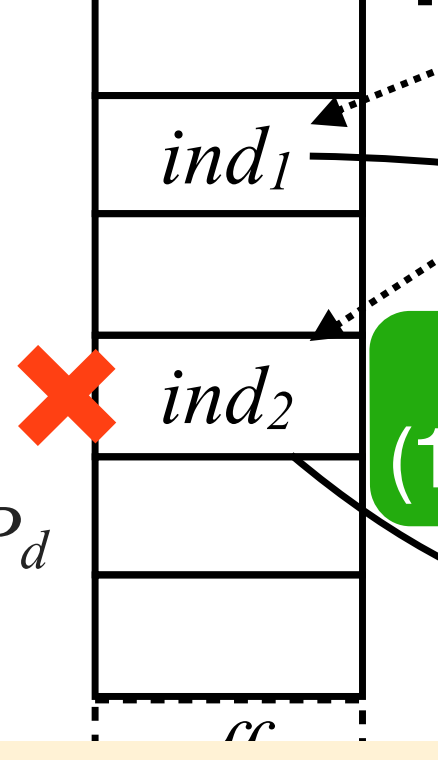

(Asynchronous)

(1) Evaluation
Archive

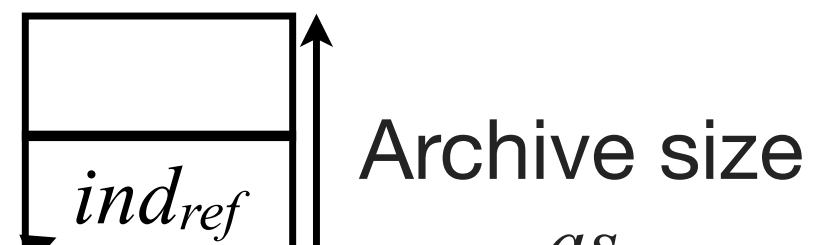

$a s$

Reference

(3) Mutation

\& Crossover

Deletion based on comparison with reference

$\rightarrow$ delete bad individuals

Evaluated

(2) Selection

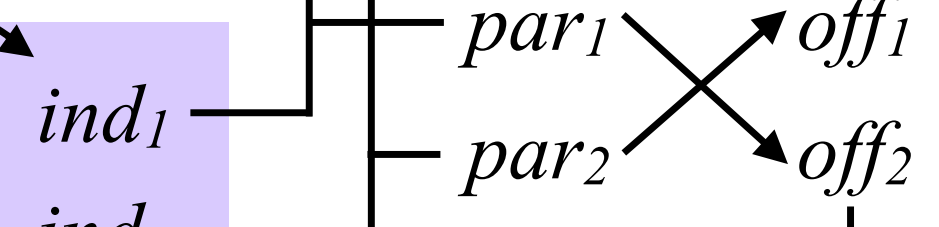
ind $_{2}$ 2

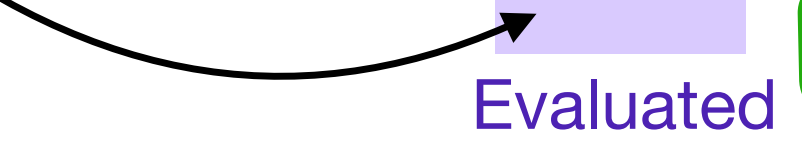

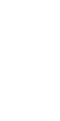




\section{Proposed method ARE-EA}

(5) Fitness deletion
(6) Reaper deletion
(6) Reaper deletion individuals with long evaluation time is deleted

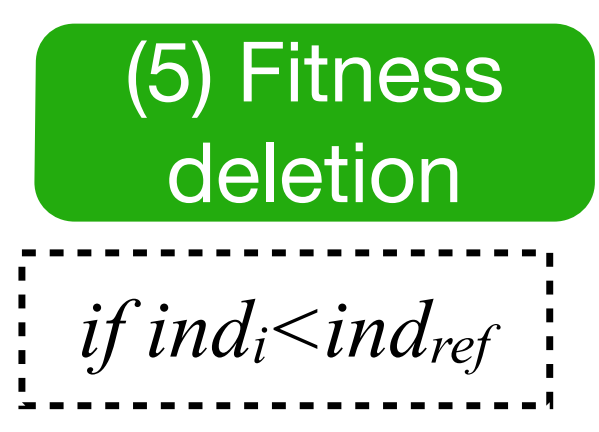
with probability $P_{d}$

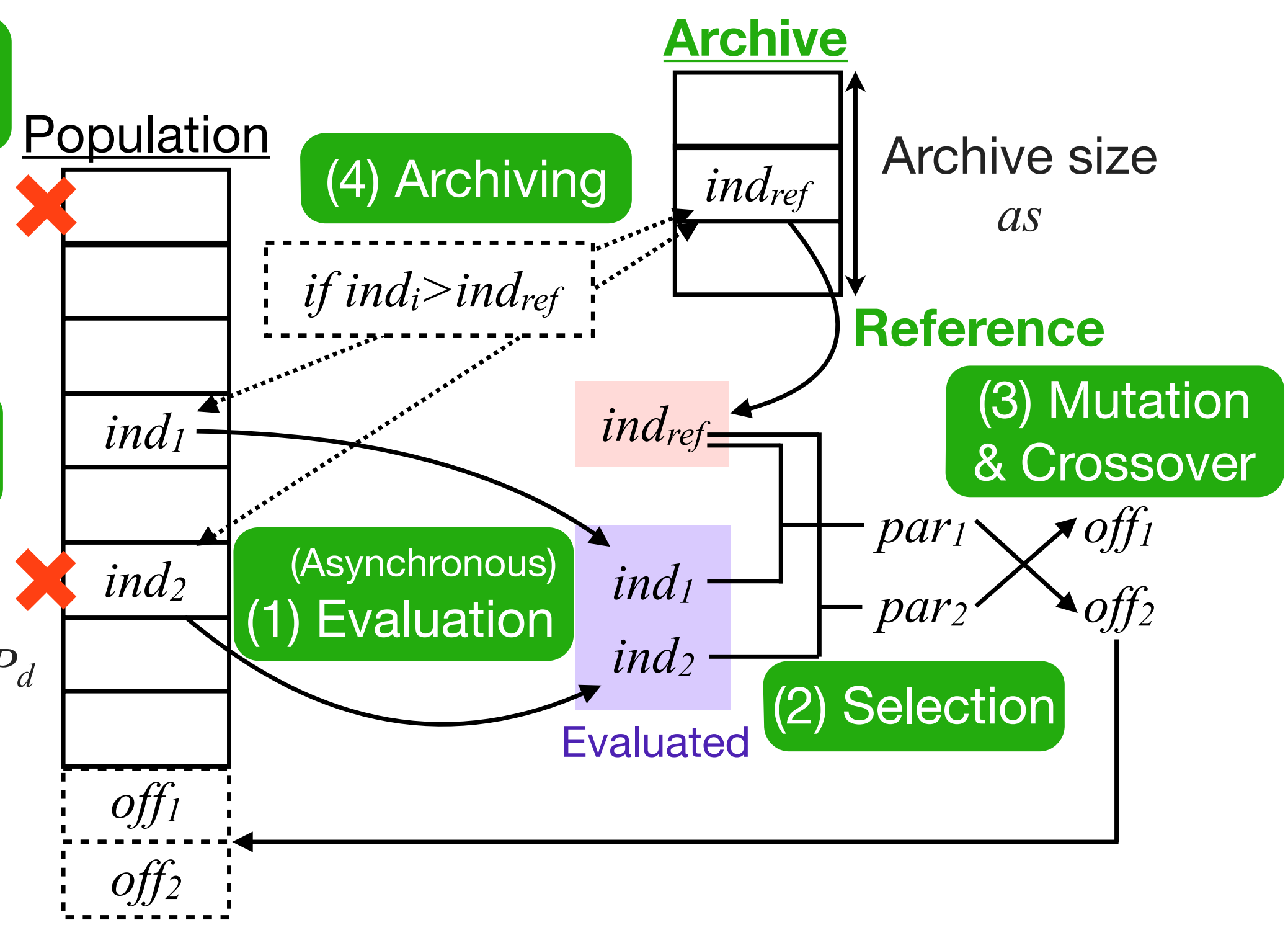




\section{Experiment}

\section{Comparison}

\section{ARE-GP vs. $(\mu+\lambda)$-GP}

(asynchronous) (synchronous)

in situation where evaluation times are excessively different

\section{Testbed problem}

Employing Linear GP (LGP) testbeds

Symbolic regression

$1 f(x)=x^{4}+x^{3}+x^{2}+x$

$2 f(x)=x^{6}-2 x^{4}+x^{2}$

$3 f(x)=\sin \left(x^{2}\right) \times \cos (x)-1$

$4 f(x)=\ln (x+1)+\ln \left(x^{2}+1\right)$

[J. McDermott, et al., 2012]
Instruction set

,,$+- \times$, /, sin, cos, exp, ln

$x_{0} \ldots x_{7}$, constant $=\{1,2, \ldots, 9\}$ 


\section{Settings}

Different evaluation time situations

(1) Different computing speed (e.g., Difference of processing ability)

\section{Same}

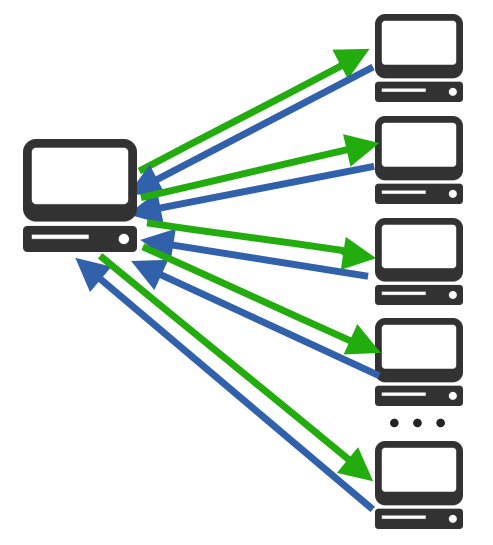

100insts./unit time 100insts./unit time 100insts./unit time 100insts./unit time 100insts./unit time
Different

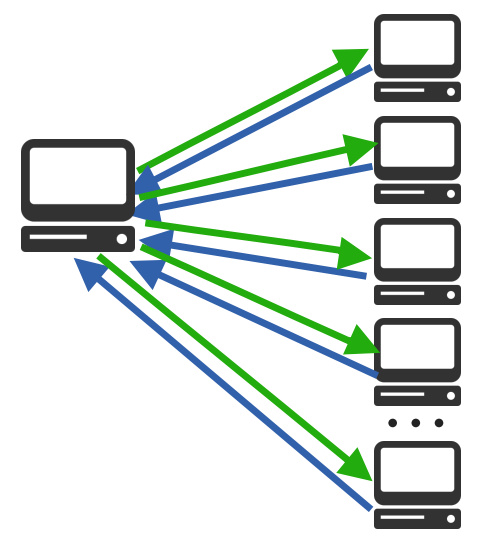

100insts./unit time 80insts./unit time 60insts./unit time 40insts./unit time 20insts./unit time

(2) Evaluation failure (e.g., Infinite loop, Communication error)

No failure

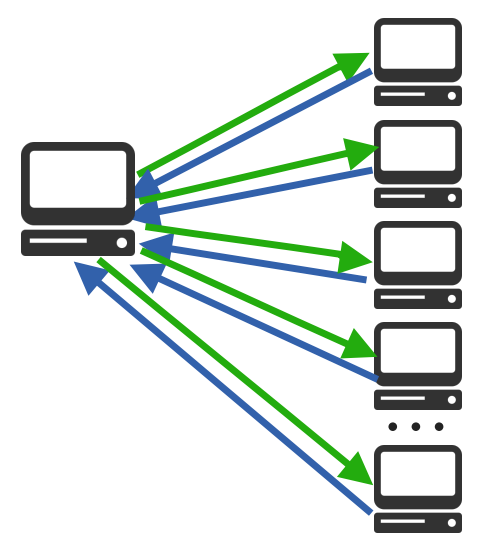

Failure

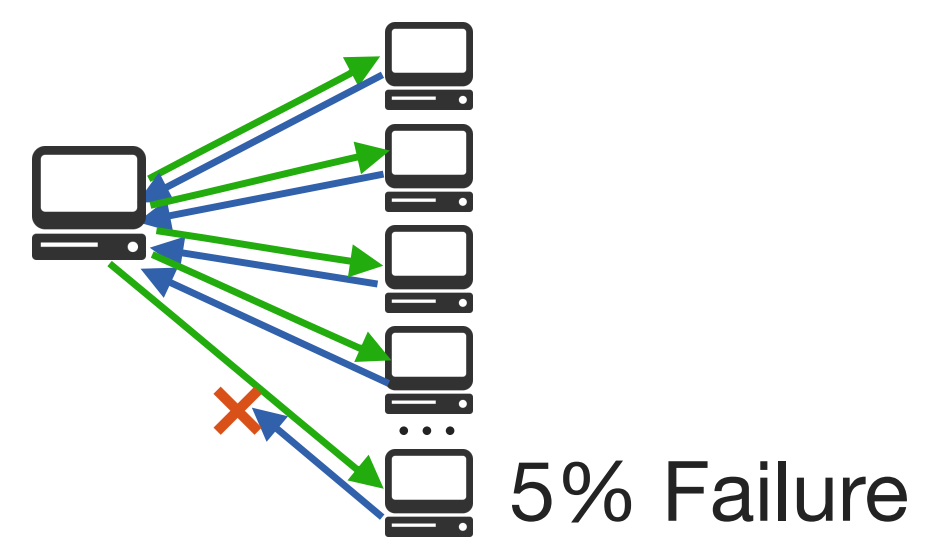




\section{Cases}

\begin{tabular}{|c|c|c|}
\hline Failure Speed & Same & Different \\
\hline No failure & Case1 & Case2 \\
\hline Failure & Case3 & Case4 \\
\hline
\end{tabular}

${ }^{*}(\mu+\lambda)$-GP uses ideal limitation time to cut off evaluations in Cases $3 \& 4$ *ARE-GP : archive size $a s=5$, deletion probability $P_{d}=0.5$

\section{Evaluation Criterion 20 trials}

Average fitness according to the same elapsed unit time

$$
\text { fitness }=\frac{1}{m} \sum_{i=1}^{m}\left(\hat{y}_{i}-y_{i}^{*}\right)^{2} \begin{aligned}
& m: \text { \# of test data } \\
& \hat{y}_{i}: \text { output } \quad y_{i}^{*}: \text { target value }
\end{aligned}
$$




\begin{tabular}{|c|c|c|}
\hline Failure Speed & Same & Different \\
\hline No failure & Case1 & Case2 \\
\hline Failure & Case3 & Case4 \\
\hline
\end{tabular}




\section{Result : Case1}

0.3

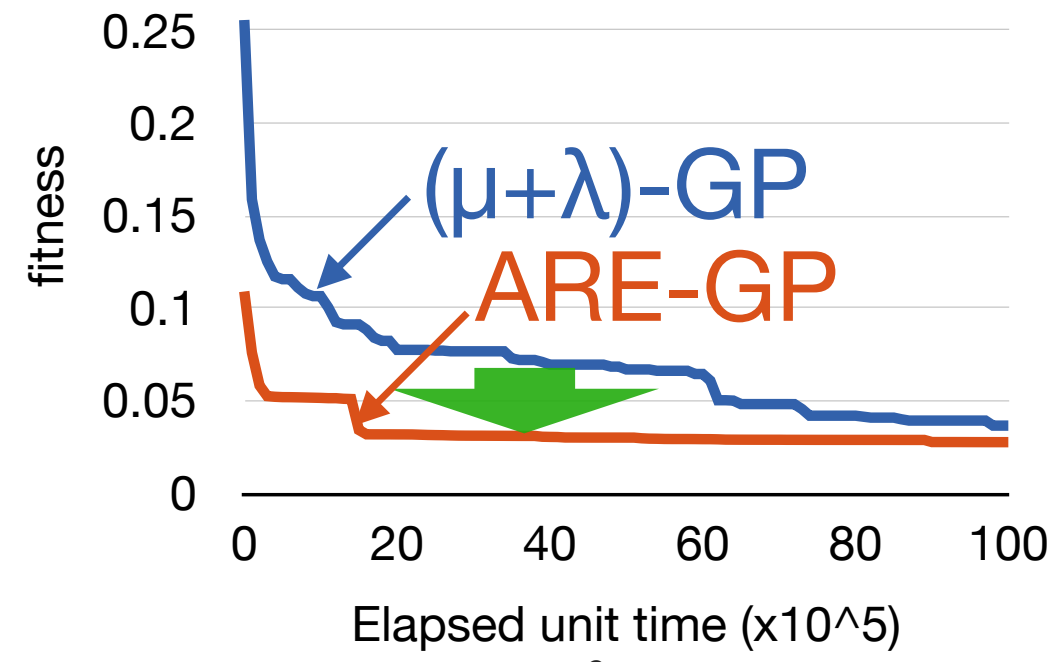

(3) $f(x)=\sin \left(x^{2}\right) \times \cos (x)-1$

0.015

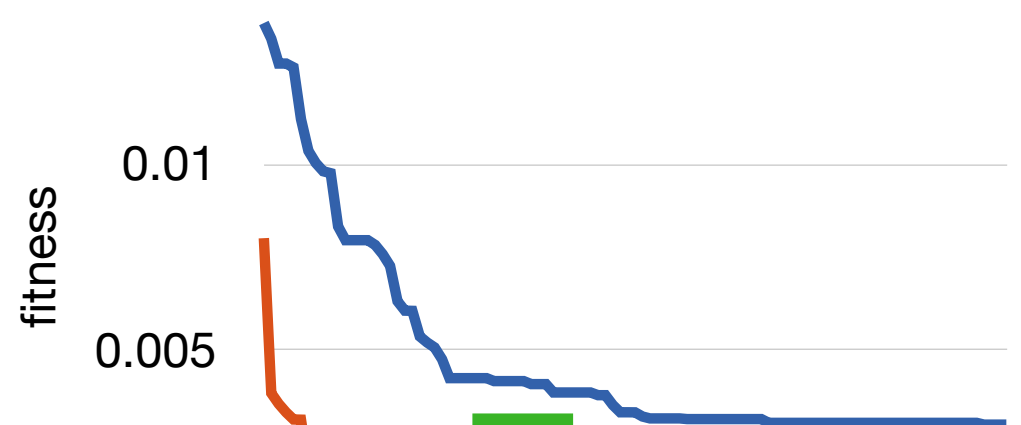

\section{(2) $f(x)=x^{6}-2 x^{4}+x^{2}$}

0.003
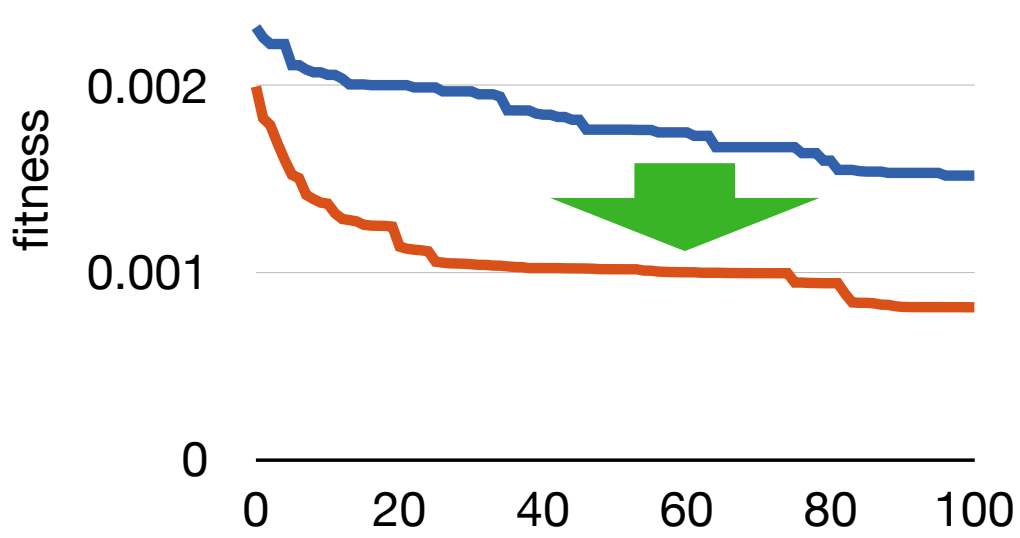

Elapsed unit time $\left(x 10^{\wedge} 5\right)$

(4) $f(x)=\ln (x+1)+\ln \left(x^{2}+1\right)$

0.1

0.08

$\begin{array}{ll}\stackrel{\infty}{\mathscr{\Phi}} & 0.06 \\ \stackrel{5}{*} & 0.04\end{array}$

חח

ARE-GP $>(\mu+\lambda)-\mathrm{GP}$

$\rightarrow$ ARE-GP evolves individuals without waisting time 


\begin{tabular}{|c|c|c|}
\hline Failure Speed & Same & Different \\
\hline No failure & Case1 & Case2 \\
\hline Failure & Case3 & Case4 \\
\hline
\end{tabular}




\section{Result : Case4}

\begin{tabular}{|c|c|c|}
\hline $\begin{array}{c}\text { Speed } \\
\text { Failure }\end{array}$ & Same & Different \\
\hline No failure & Case1 & Case2 \\
\hline Failure & Case3 & Case4 \\
\hline
\end{tabular}

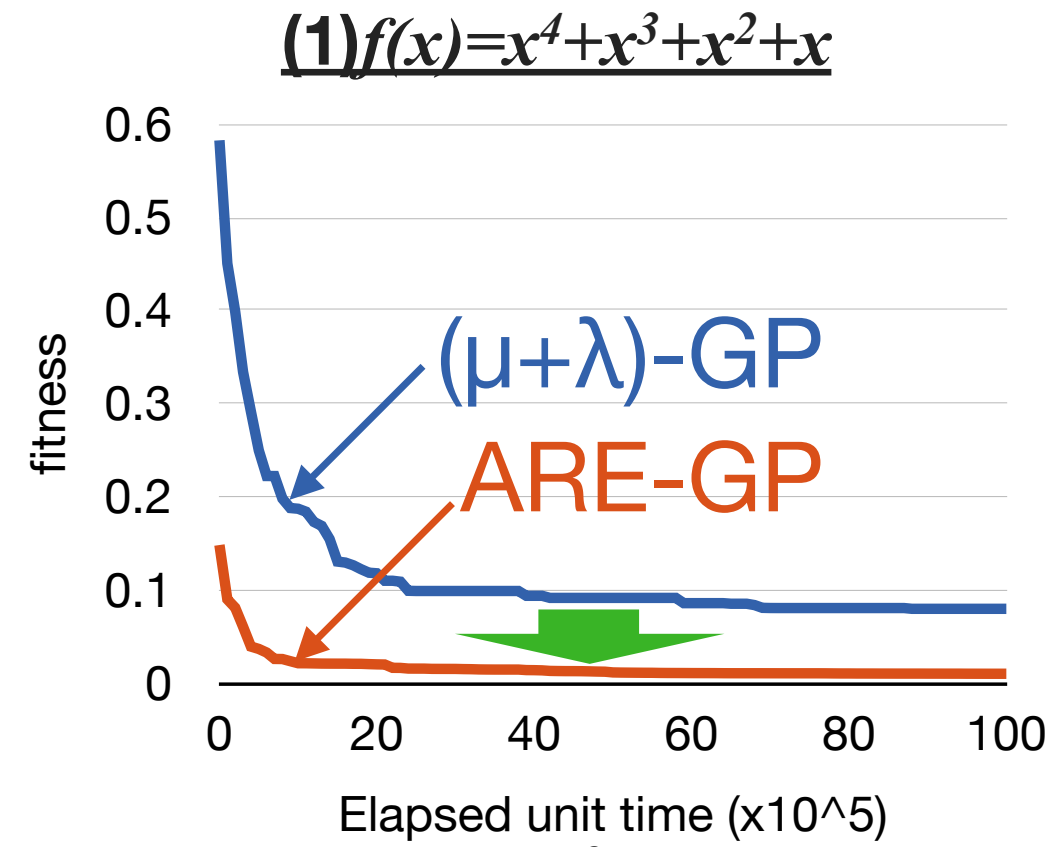

(3) $f(x)=\sin \left(x^{2}\right) \times \cos (x)-1$
(2) $f(x)=x^{6}-2 x^{4}+x^{2}$

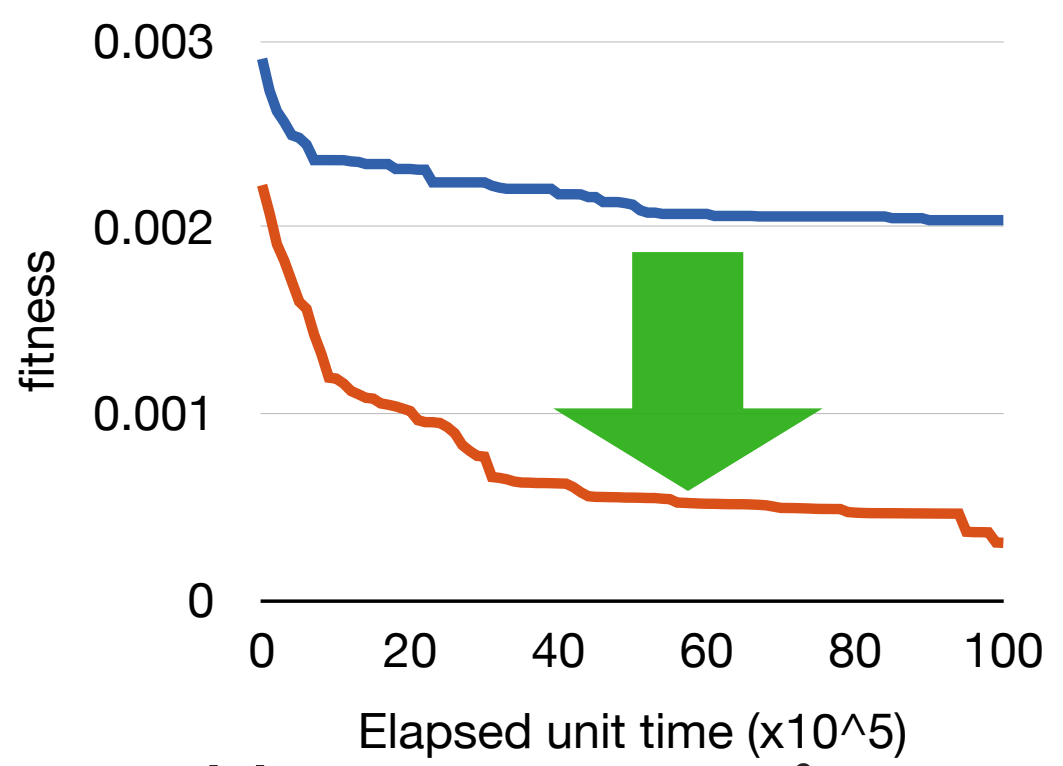

(4) $f(x)=\ln (x+1)+\ln \left(x^{2}+1\right)$

0015

ARE-GP evolves individuals without any limitations

ARE-GP> $>(\mu+\lambda)-G P$

ARE-GP efficiently evolves individuals in situation where evaluation times are excessively different 


\section{Result}

\section{ARE-GP $>(\mu+\lambda)$-GP in all cases and in all testbeds}

ARE-GP only uses a few evaluations

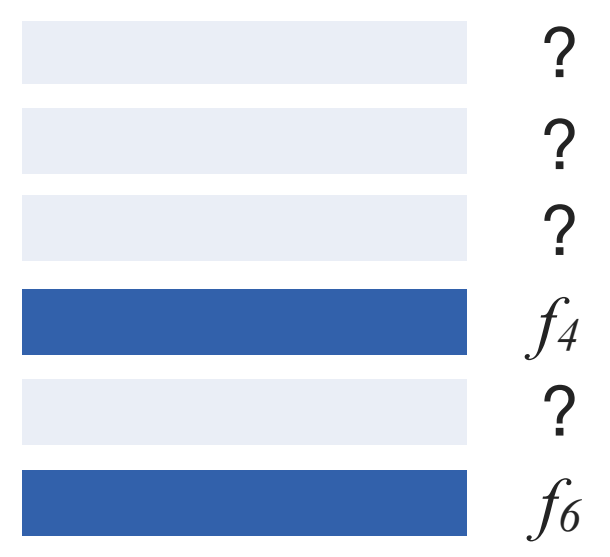

$(\mu+\lambda)$-GP

can use all evaluations

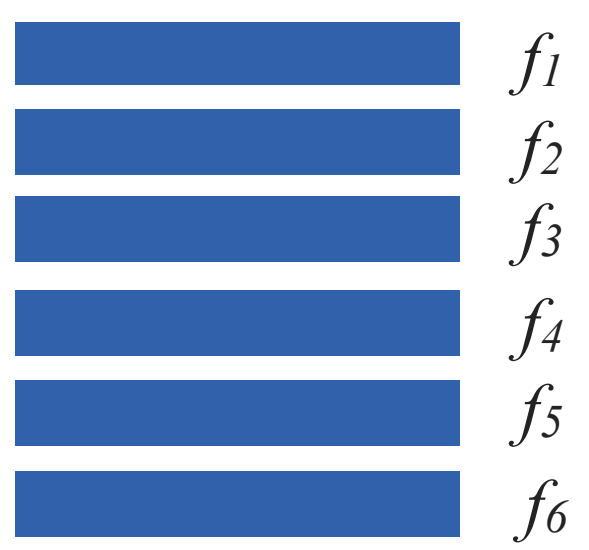




\section{Comparison of Case1\&Case4}

Ratio of fitness between Case 1 and Case 4

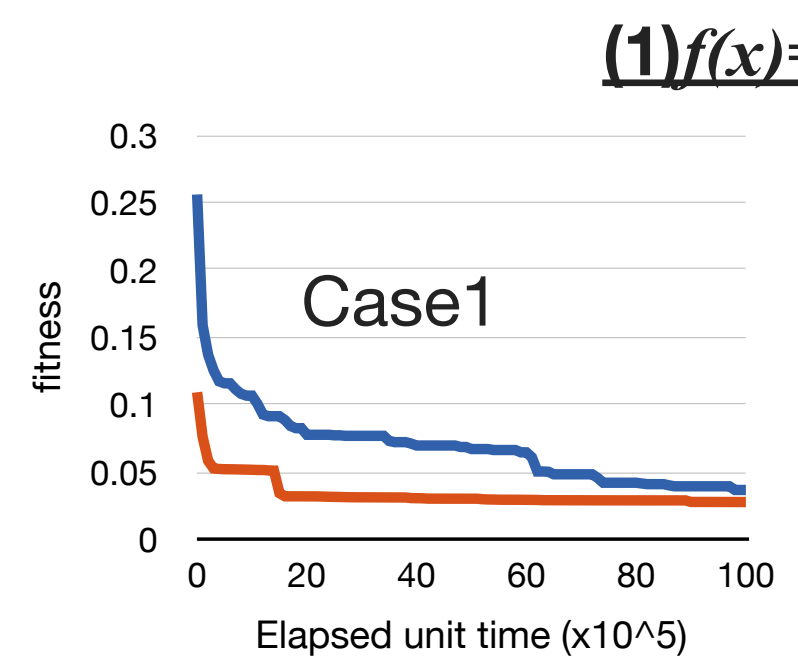

(1) $f(x)=x^{4}+x^{3}+x^{2}+x$
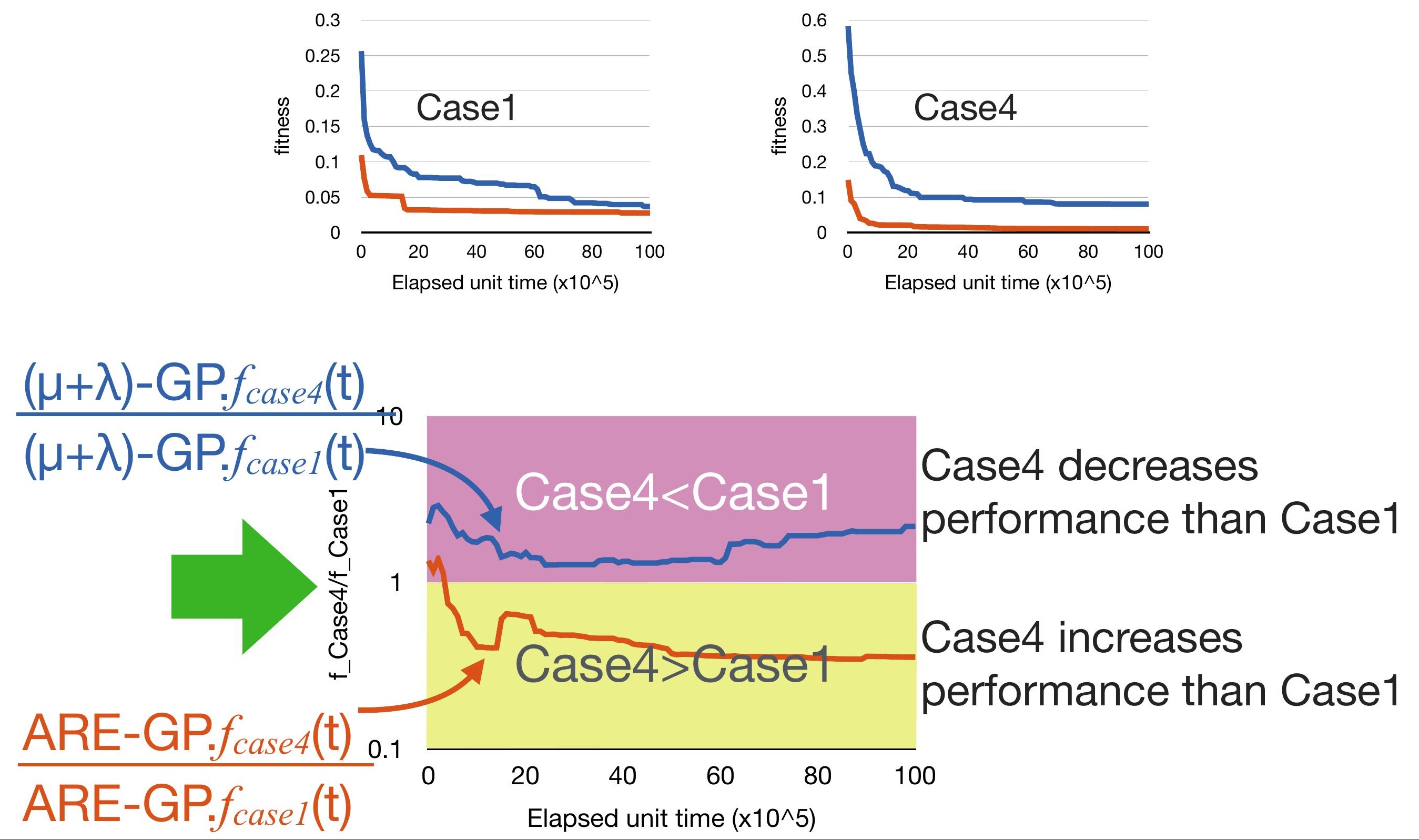


\section{Comparison of Case1\&Case4}

(1) $f(x)=x^{4}+x^{3}+x^{2}+x$

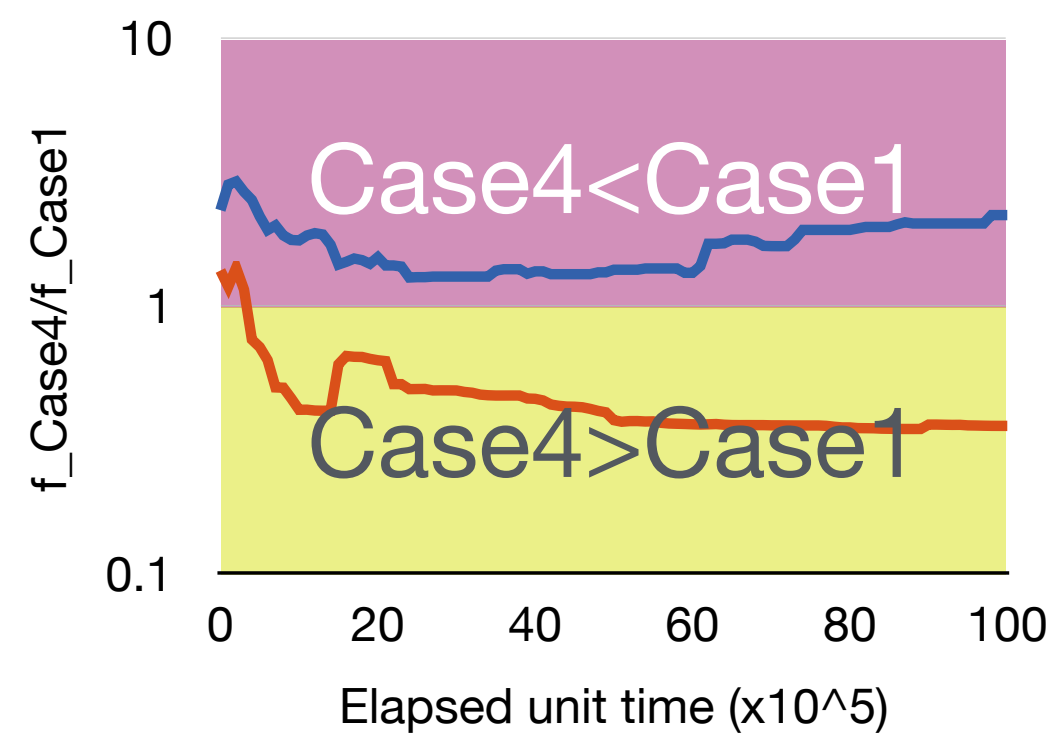

(3) $f(x)=\sin \left(x^{2}\right) \times \cos (x)-1$

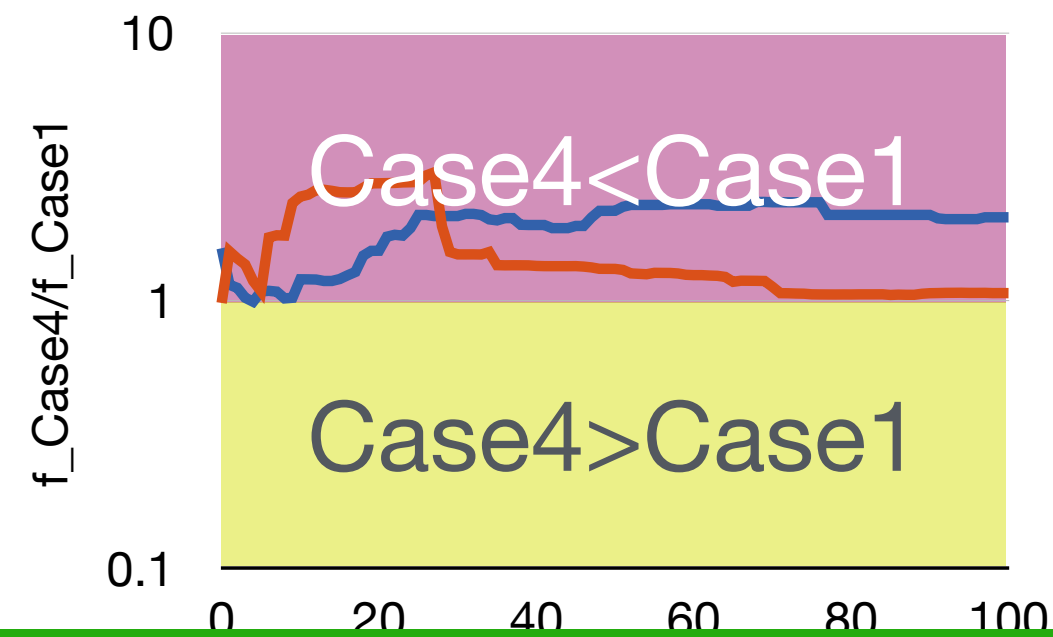

(2) $f(x)=x^{6}-2 x^{4}+x^{2}$

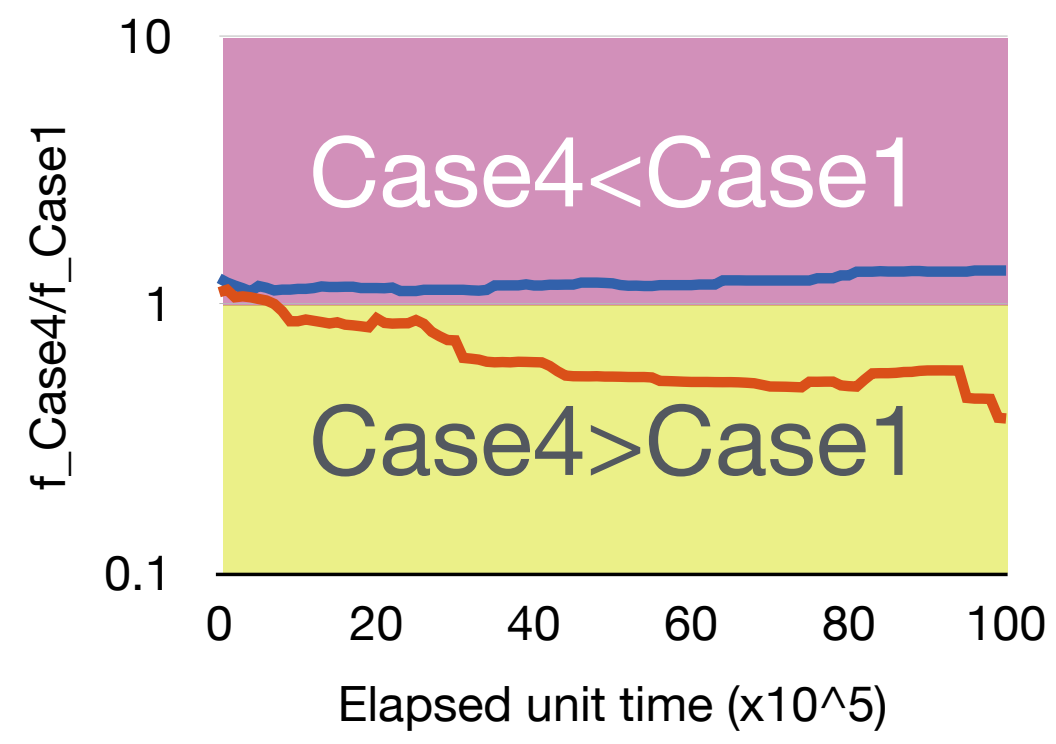

(4) $f(x)=\ln (x+1)+\ln \left(x^{2}+1\right)$

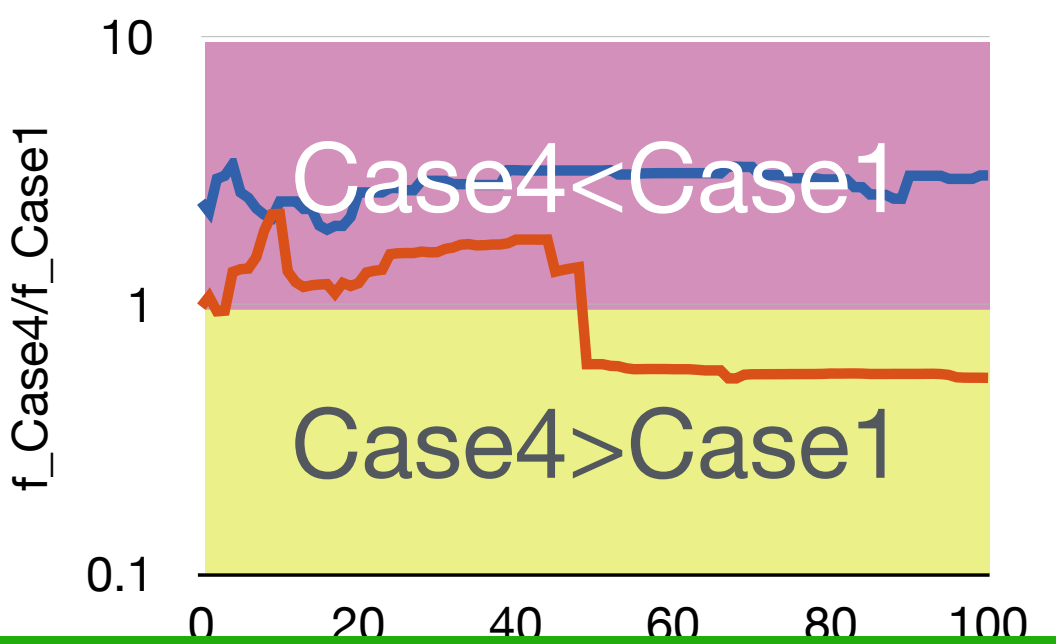

ARE-GP has possibility to improve search performance in different evaluation time 


\section{Conclusion}

Objective

- Proposing EA using Asynchronous Reference-based Evaluation (ARE-EA)

- Investigating effectiveness of ARE-EA in excessively different evaluation times

- different computing speed

- evaluation failure

Implications

- ARE-GP $>(\mu+\lambda)-G P$

- in excessively different evaluation time

- ARE-GP improves performance in different evaluation time Future works

- Validation in parallel computing environment

- Adaptation of parameters $P_{d}$ and as 